\title{
Metas Parentais de Socialização sobre Emoções: Um Estudo Exploratório
}

\section{Parent's Goals of Socialization about Emotions: An Exploratory Study}

\section{Metas Parentales de Socialización de Emociones: Un Estudio Exploratorio}

\section{Deise Maria Leal Fernandes Mendes*}

Universidade do Estado do Rio de Janeiro - UERJ, Rio de Janeiro, Rio de Janeiro, Brasil

\author{
Joana Lezan Sant'Anna** \\ Universidade do Estado do Rio de Janeiro - UERJ, Rio de Janeiro, Rio de Janeiro, \\ Brasil
}

Dandara de Oliveira Ramos***

Instituto Gonçalo Moniz - Fiocruz-Bahia, Salvador, Bahia, Brasil

\begin{abstract}
RESUMO
Estudar metas parentais de socialização emocional permite conhecer particularidades de contextos socioculturais e seus impactos no desenvolvimento infantil. Para analisar essas metas em mães e pais e sua relação com a valorização da autonomia ou da relação, realizou-se um estudo exploratório em que foram entrevistadas 60 duplas mãe-pai de filhos com até três anos, residentes na cidade do Rio de Janeiro. As respostas das entrevistas foram analisadas por uma técnica de análise de conteúdo e, posteriormente, foram calculadas frequências de evocações para as categorias identificadas e empregados procedimentos estatísticos descritivos e testes de associação entre grupos. Os resultados indicaram que as características emocionais desejadas por mães e pais para os filhos quandoadultos sinalizavam valorização da autonomia, independência e autoconfiança emocional, com as condições para o seu desenvolvimento estando principalmente centradas no contexto. A atuação parental prevalente foi a de educar/orientar para o desenvolvimento das metas emocionais que estabeleceram. Não foram encontradas diferenças significativas entre mães e pais, nem entre mães-pais de meninos e meninas quanto a quaisquer dos aspectos investigados. Sugere-se novos estudos abarcando distintas configurações familiares, com participantes de variados níveis de escolaridade e em outros contextos socioculturais.
\end{abstract}

Palavras-chave: metas de socialização, desenvolvimento emocional, parentalidade, autonomia. 


\begin{abstract}
Studying parental socialization goals allows us to understand the characteristics of sociocultural contexts and its impacts on child development. In order to analyze such goals in mothers and fathers and its relationship with their values regarding autonomy and relatedness, an exploratory study was conducted in which we interviewed 60 mother-father couples of children up to three years of age, living in the city of Rio de Janeiro. Interview responses were analyzed by a content analysis technique and, subsequently, emission frequencies were calculated for the identified categories and descriptive statistical procedures and association tests between groups were employed. Results showed that the emotional characteristics that the parents desired for their children as adults valued autonomy, independence and emotional self-confidence, with the conditions for child development mostly centered in contextual factors. The most frequently reported parental behavior was involvement in education/guidance towards their child developmental goals. No differences were found between mothers and fathers, neither between parents of girls or boys regarding any of the variables analyzed. Further studies are suggested covering different family configurations, with participants of varying levels of education and in other socio-cultural contexts.
\end{abstract}

Keywords: socialization goals, emotional development, parenting, autonomy.

\title{
RESUMEN
}

Estudiar metas parentales de socialización emocional permite conocer particularidades de contextos socioculturales y sus impactos en el desarrollo infantil. Para analizar esas metas en madres y padres y su relación con la valorización de la autonomía o de la relación, se realizó un estudio exploratorio y se entrevistó 60 dobles madre-padre de hijos con hasta tres años, residentes en la ciudad de Río de Janeiro. Las respuestas de la entrevista se analizaron mediante técnica de análisis de contenido $y$, posteriormente, se calcularon las frecuencias de emisión para las categorías identificadas y se emplearon procedimientos estadísticos descriptivos y pruebas de asociación entre grupos. Los resultados indicaron que las características emocionales deseadas por madres y padres para los hijos cuando adultos señalaban valorización de autonomía, independencia y autoconfianza emocional, con las condiciones para su desarrollo estando principalmente centradas en el contexto. La actuación parental prevalente fue la de educar/orientar para el desarrollo de las metas emocionales que establecieron. No se encontraron diferencias significativas entre madres y padres, ni entre madres-padres de niños y niñas en cuanto a cualquiera de los aspectos investigados. Se sugieren estudios adicionales que abarquen diferentes configuraciones familiares, participantes de diferentes niveles de educación y en otros contextos socioculturales.

Palabras-clave: objetivos de socialización, desarrollo emocional, crianza de los hijos, autonomía.

Metas de socialização se referem a ideais ou objetivos dos pais sobre de que modo desejam que seus filhos sejam no futuro e aquilo que eles esperam que sua criação ajude a promover em seus filhos, podendo ser também designadas como metas de desenvolvimento (Keller \& Kärtner, 2013). Elas capturam princípios e ideias do grupo 
cultural com relação ao que priorizam como modo ideal de ser, dentro de um ambiente sociocultural particular. Tais metas de mães e pais são parte de sistemas de crenças parentais e associam-se a práticas de cuidado e educativas, estando relacionadas a trajetórias de desenvolvimento dos filhos.

Diversos estudos têm sido conduzidos nas últimas décadas com o propósito de avaliar metas de socialização em diferentes contextos culturais (Harwood, Schölmerich, Ventura-Cook, Schulze, \& Wilson, 1996; Leyendecker, Lamb, Harwood, \& Shölmerich, 2002; Park, Coello, \& Lau, 2014; Raval, Raval, \& Deo, 2014). Os estudiosos do tema entendem que os objetivos dos pais em relação ao processo de socialização dos filhos a médio e longo prazos, bem como suas avaliações do comportamento infantil, são influenciados culturalmente (Otto \& Keller, 2016; Tamm, Kasearu, Tulviste, \& Trommsdorff, 2016). Assim é que, no que concerne às necessidades humanas de autonomia e relação interpessoal (Kağitçibaşi, 2007), há culturas em que os pais tendem a desejar que seus filhos atinjam elevado grau de autonomia, enquanto em outras, predominam metas de desenvolvimento marcadas pela forte interdependência e ligação ao grupo social e aos demais indivíduos que o constituem (Keller, 2016; Keller \& Kärtner, 2013).

Um sistema de crenças parentais mais individualista, de acordo com Leyendecker et al. (2002), se relaciona a uma valorização maior da independência e autonomia na criança, refletida, no estudo que empreenderam, em uma maior frequência de respostas nas categorias definidas pelos autores como autoaperfeiçoamento e autocontrole. Entendem ainda que um sistema de crenças mais sociocêntrico aponta para uma valorização maior da interdependência e relação interpessoal, associada à priorização de ideias refletidas nas categorias definidas como expectativas sociais e bom comportamento. Argumentam que é possível pensar-se na existência de certa coesão intra-grupal quanto às metas de socialização em determinado grupo ou sociedade, mas advertem que isto não significa dizer que não haverá diversidade ou traços de heterogeneidade. De fato, a análise dos objetivos de longo prazo declarados pelas participantes do estudo, mães que migraram de países da América Central e mães americanas de origem europeia, levaram a resultados que foram interpretados pelos autores como indicativos da necessidade de se estudar não só as variações entre os grupos, mas as intra-grupais, ao se tratar os construtos do individualismo e do sociocentrismo.

Destaca-se também a importância de ser levado em conta, de forma especial, o ambiente sociocultural em que as pessoas vivem e se desenvolvem, além de sua cultura de origem. Mães turcas, habitantes da Austrália, como relatado em Sen, Yavuz-Muren e Yagmurlu (2014), foram indagadas a respeito de suas metas de socialização e 
práticas de cuidado. Uma parte das mães priorizou um modelo cultural mais individualista, considerado o mais marcante na Austrália, favorecendo, portanto, a autonomia de seus filhos, enquanto outras pareceram valorizar um modelo cultural mais sociocêntrico, característico da Turquia, em que é desejada uma trajetória de desenvolvimento relacional (Kağitçibaşi, 2007).

Procurando caracterizar metas de socialização de cuidadores, especialmente mães e pais, em contextos brasileiros diversos, estudos indicaram que tanto a autonomia quanto a relação são valorizadas (Seidl-de-Moura et al., 2008, 2009; Vieira et al., 2010). Em pesquisa realizada por Seidl-de-Moura et. al. (2008) com 350 mães de sete cidades de todas as cinco regiões geográficas brasileiras, a comparação entre médias mostrou que as mães deram mais ênfase à automaximização (autoaperfeiçoamento) e ao comportamento adequado do que ao conteúdo representado pelas outras categorias, apresentando um padrão que promove 0 desenvolvimento de uma autonomia-relacionada nas crianças. Foi encontrada, contudo, variação intracultural nos objetivos de socialização no que concerne às diferentes cidades estudadas, atribuída a variáveis sociodemográficas, como o tamanho da cidade e o nível educacional das mães.

Ao serem investigadas apenas mães do Rio de Janeiro, Seidl-deMoura et al. (2009) encontraram resultados que confirmavam os do estudo anterior. As mães mostraram tendência à valorização da autonomia, mas a autonomia não era mencionada pelo grupo de entrevistadas sem que também manifestassem claras preocupações de natureza sociocêntrica. As análises não indicaram diferença estatisticamente significativa nos escores das duas categorias mais frequentemente mencionadas, sendo uma associada à autonomia e a outra à relação.

No estudo de Vieira et al. (2010), 600 mães participaram, sendo metade delas residentes em cidades pequenas e a outra metade em capitais brasileiras. Observou-se preponderância de metas de socialização relacionais nas cidades interioranas e de metas tanto de autonomia quanto de relação, em capitais. Neste estudo, o nível educacional não foi considerado um fator que tenha impactado as metas de socialização. Para os autores, a maior urbanização está associada a um aumento da competitividade, que diminui os valores ligados ao grupo e aumenta os valores de autonomia, ligados ao indivíduo e suas prioridades.

No âmbito das metas de socialização, foram objeto de interesse nesse estudo as metas de socialização voltadas para as emoções. Justificase a relevância de investigações com essa preocupação por razões da mesma natureza daquelas que sustentam a importância dos estudos de metas de socialização de caráter mais geral. Assim como argumenta-se a mediação da cultura e de um sistema de crenças 
parentais, a que as metas de desenvolvimento estão vinculadas, no desenvolvimento da criança, pode-se pensar de modo análogo quanto ao que mães e pais desejam para seus filhos no domínio específico do desenvolvimento emocional. Muito embora seja uma temática que parece ter grande relevância teórica e prática, há total carência de publicações nacionais com esse foco.

Mendes, Pessôa e Cavalcante (2016) discutem a necessidade de pesquisas que, ampliando esse interesse, façam ainda uma articulação com a valorização da autonomia e/ou da relação pelos pais e demais cuidadores da criança. Nesse sentido, pesquisas denotam uma associação entre metas e concepções de self-mais autônomos, mais relacionais, ou balanceados- de mães e tendências na valorização de metas de socialização emocional. Mães chinesas de Hong Kong demonstraram uma concepção de self predominantemente interdependente (relacional), ao mesmo tempo em que valorizaram mais as competências emocionais relacionais, em detrimento de competências emocionais individualistas (Chan, 2011). Nesse estudo, em que participaram 189 mães, a autora buscou identificar a relação entre a orientação de self defendida pelas mães e as metas de socialização emocional que possuíam para o futuro de seus filhos.

Embora as mães tenham priorizado a competência emocional relacional, também enfatizaram a competência emocional individualista como meta de desenvolvimento dos filhos, o que Chan (2011) discute considerando um conjunto de mudanças socioeconômicas marcantes. Em especial, menciona o aumento no número de anos de escolarização gratuita e obrigatória e o sistema econômico de Hong Kong que teria passado de essencialmente agrícola para uma economia centrada no setor terciário. Desse modo, conclui que a autonomia se tornou gradualmente uma espécie de "bem pessoal", sendo cada vez mais valorizada.

A noção de competência emocional valorizada por mães com uma concepção de self independente deve ser considerada, segundo Chan (2011), como incluindo a compreensão das emoções, sua expressão de modo natural, especialmente as voltadas para o eu, estando associada a uma competência emocional independente ou individualista. De modo diverso, a noção de self interdependente é entendida como vinculada à importância de se aprender a regular as emoções, especialmente as centradas no eu, e de incentivaras que promovem harmonia no grupo social, priorizando uma competência emocional relacional.

Mais amplamente, foi visto que as metas de socialização se vinculam a estratégias de socialização emocional e modos de expressões de emoções, em culturas diversas. Foi observada, por exemplo, diferença marcante na expressão de emoções em dois ambientes prototípicos: famílias da classe média urbana ocidental (famílias 
alemãs de Berlim) e famílias de agricultores em área rural em sociedades não-ocidentais (o grupo dos Nso, do noroeste de Camarões). No primeiro ambiente, houve uma valorização maior de metas de socialização voltadas para a autonomia e no segundo de metas voltadas para a relação (Keller, 2016; Keller \& Otto, 2009).

Keller e Otto (2009) relataram ainda que a expressão de emoções é tratada diferentemente nesses contextos. Enquanto as mães Nso revelaram estratégias de socialização voltadas ao controle emocional inicial, com especial ênfase na supressão de emoções consideradas negativas, como a tristeza e a raiva, as alemãs enfatizaram a expressão de emoções, favorecendo especialmente a emotividade considerada positiva, como a alegria. As autoras discutiram que, na cultura Nso, a relação é uma força organizadora do desenvolvimento, promovendo o controle emocional inicial, enquanto na cultura alemã, o organizador do desenvolvimento é a autonomia, promovendo a expressividade emocional.

Fonseca, Cavalcante e Mendes (2017) entrevistaram mães brasileiras em um contexto rural no interior do estado do Pará a respeito de suas metas de socialização de emoções. Os resultados revelaram que as mães priorizaram metas relacionadas à automaximização e chamavam para si a condição necessária para que a criança desenvolvesse as características almejadas. As autoras adotaram a noção de metas de automaximização como sendo as que envolvem preocupação com que a criança se torne emocionalmente autoconfiante e independente, e que desenvolva plenamente suas capacidades ligadas à esfera emocional. Quando questionadas sobre as condições para o desenvolvimento das características emocionais que almejavam para os filhos, preponderaram as respostas com condições centradas na mãe (ou cuidador primário), ou seja, da mãe sendo modelo, orientando e ensinando por demonstração ou participação.

No que se refere à atuação parental para o desenvolvimento de características emocionais desejadas, a maioria das participantes destacou a importância de educar e orientar, oferecendo modelos, disciplinando, aconselhando, ou ensinando por explicações verbais ou medidas disciplinares. Segundo as autoras, embora fosse esperada uma associação das características sociodemográficas do grupo estudado com a adoção de uma orientação relacional, as mães majoritariamente mencionaram metas de automaximização, que se referem de modo mais direto a características de um indivíduo autoconfiante na manifestação e na vivência das emoções e sentimentos, características estas relacionadas a um modelo de orientação autônoma.

Estudar metas parentais de socialização permite conhecer melhor particularidades de contextos socioculturais e seus impactos no desenvolvimento de crianças. Estudar metas parentais de socialização 
ligadas às emoções é algo ainda pouco investigado e permite que se explore as intencionalidades de mães e pais a respeito de aspectos centrais do desenvolvimento emocional dos filhos, e seu impacto no desenvolvimento destes, entendendo essa dinâmica como mediada pela cultura. Nesse sentido, buscou-se, nesse estudo exploratório, conhecer as metas de socialização de emoção de mães e pais de um contexto urbano brasileiro, a cidade do Rio de Janeiro, articulando-as à valorização da autonomia e/ou relação. Com isto, espera-se contribuir para suprir lacuna na literatura, especialmente a brasileira, em que há ampla carência de pesquisas com esse objetivo.

\section{Método}

\section{Participantes}

A amostra foi composta por 60 duplas de mães e pais de crianças com até três anos de idade. Como critérios de inclusão, além da idade da criança dentro do limite estabelecido, foi requerido que os pais vivessem em união estável, vivendo na mesma casa. Eram todos residentes na cidade do Rio de Janeiro. A média de idade das mães foi de 31,7 anos $(D P=5,46)$ e dos pais de 34,6 anos $(D P=5,3)$. Quanto ao nível de escolaridade, $7 \%$ das mães tinham até o Ensino Fundamental (EF) completo, $22 \%$ até o Ensino Médio (EM) completo e $72 \%$ até o Ensino Superior (ES) completo ou Pós-graduação. Dos pais, $7 \%$ tinham até o EF completo, $25 \%$ até o EM completo, e $68 \%$ até o ES completo ou Pós-graduação.

\section{Instrumentos}

Como instrumentos foram utilizados o questionário de dados sociodemográficos, com informações como a idade do respondente e de seu filho ou filha, escolaridade, estado civil, e sexo da criança; e o "Questionário sobre metas parentais de socialização da emoção", desenvolvido para o projeto "Expectativas de desenvolvimento emocional e metas parentais de socialização das emoções em crianças", coordenado pela primeira autora, e que deu origem aos dados apresentados no presente estudo. Deste último questionário, foram aqui consideradas três questões abertas. São elas: "Que características emocionais você desejaria que seu filho(a) tivesse como adulto? ", "O que você acha que é necessário para que ele(a) desenvolva/tenha essas características? " e "O que você pensa que pode fazer para que ele(a) possa desenvolver/ter essas características?". Para fins de concisão textual, as respostas a estas perguntas serão referidas ao longo do manuscrito como 
características desejadas, condições para o desenvolvimento e atuação parental, respectivamente.

\section{Procedimentos de coleta e análise de dados}

O grupo de participantes desse estudo foi constituído a partir de indicações, por meio da técnica bola de neve (snowball), por integrantes do grupo de pesquisas de que fazem parte as autoras e participantes de outros estudos realizados. Após contato inicial, em que eram apresentados os objetivos do estudo e outros pontos principais que constam do Termo de Consentimento Livre e Esclarecido, foi agendado encontro em local, data e horário da preferência dos participantes para que o referido termo fosse assinado e os instrumentos aplicados. A aplicação foi realizada por membros da equipe, estudantes de Psicologia, treinados para esse fim, sendo prevalente como local da coleta a residência dos participantes. No início do encontro, os objetivos e procedimentos da coleta de dados foram lembrados aos participantes que passaram à assinatura do TCLE. Em seguida, foi solicitado que fossem preenchidos os dois questionários previstos. O preenchimento dos instrumentos foi feito pelo próprio participante. Quando estavam presentes no mesmo encontro a mãe e o pai da criança, o preenchimento foi sempre feito de modo independente, sem que um tomasse conhecimento das respostas do outro. Foram seguidos os procedimentos éticos para pesquisas com seres humanos, segundo as normas da Comissão Nacional de Ética em Pesquisa (CONEP) e do Conselho Nacional de Saúde, tendo o estudo sido aprovado pelo Comitê de Ética da Universidade do Estado do Rio de Janeiro (Protocolo 010.3.2010).

O tratamento escolhido para análise da segunda e terceira questões do "Questionário sobre metas parentais de socialização da emoção" foi a análise de conteúdo temático-categorial (Oliveira, 2008), seguindo a identificação de unidades temáticas e de categorias nas unidades de registro, definidas a posteriori, a partir da leitura do texto. Para analisar as respostas à primeira pergunta foram definidas categorias adaptadas das utilizadas por Seidl-de-Moura et al. (2008), para estudo com população brasileira, que se basearam nas definidas por Leyendecker et al. (2002). As categorias pré-definidas usadas nesse estudo foram:

1. Automaximização - meta de que a criança se torne emocionalmente autoconfiante e independente, e que desenvolva plenamente as capacidades emocionais (ex: "que saiba expressar suas emoções de forma satisfatória e autoconfiante", "que seja emocionalmente segura de si, que saiba lidar com as emoções", "que tenha orgulho de suas conquistas, medo do perigo"). 
2. Autocontrole - meta de que a criança desenvolva a capacidade de regulação emocional, controlando a manifestação exacerbada de emoções (ex: "que seja equilibrado"; "que não se exalte toda vez que não conseguir o que quer"; "que saiba lidar com as frustrações").

3. Emotividade - meta de que a criança desenvolva a capacidade para intimidade emocional com outros, e de iniciar e manter relacionamentos afetivos (ex: "que seja amável, meigo, empático", "se preocupe com as outras pessoas").

4. Expectativas sociais - meta de que a criança atenda a expectativas sociais quanto às regras de exibição de suas emoções e sentimentos e de reação às manifestações emocionais e comportamentos das outras pessoas (ex: "que não ria alto perto do bebê que dorme"; "que não bata no amigo que lhe pegou o brinquedo").

5. Outras possibilidades - a resposta não se aplica a nenhuma das definições de categoria estabelecidas.

6. Não sabe - não sabe ou não quis responder.

Com relação às condições necessárias para o desenvolvimento das características emocionais desejadas (condições para 0 desenvolvimento), as respostas dos pais e mães foram classificadas como: a) centradas na mãe (ou cuidador) - ser modelo ou oferecer modelos, disciplinar, aconselhar, ensinar por demonstração ou participação; b) centradas no contexto - oferecer boas oportunidades sociais; dar educação de qualidade, etc; c) centradas na criança características ou capacidades inerentes à criança; d) não se aplica não há desenvolvimento, já que as características são consideradas inatas ou fruto de maturação biológica; e) outras condições - a resposta não se aplica a nenhuma das definições de categoria estabelecidas.

Por fim, com relação à atuação parental necessária para o desenvolvimento das características emocionais desejadas (atuação parental), as categorias registradas foram: a) educar/orientar - ser modelo ou oferecer modelos, disciplinar, aconselhar, conversar, ensinar por demonstração, participação, explicações verbais, ou medidas disciplinares; b) manter relações de proximidade pessoal fazer-se afetivamente presente para a criança, acompanhar, dar amor, cuidar da criança expressando carinho e afetividade, prover ambiente familiar saudável com boas relações entre seus membros; c) prover condições materiais e sociais - oferecer boas oportunidades sociais; dar educação de qualidade, prover seu sustento, bom atendimento de saúde etc; d) não se aplica - não há o que fazer, não há desenvolvimento, já que as características são consideradas inatas ou fruto de maturação biológica; e) outras atuações - a resposta não se aplica a nenhuma das definições de categoria estabelecidas.

Posteriormente, as categorias identificadas foram registradas em um banco de dados e analisadas em procedimentos estatísticos 
descritivos e testes de associação entre grupos. Especificamente, para a análise de frequência de evocação das categorias associadas a características desejadas, condições para o desenvolvimento e atuação parental, foram calculadas frequências relativas; e, para a análise das categorias preponderantes, foram realizados testes quiquadrado de aderência. Em seguida, para a comparação das frequências de evocação das categorias das mães vs pais no geral, e entre mães $v s$ pais de meninos ou meninas, foram realizados teste qui-quadrado de associação.

As respostas dos participantes para as questões abertas foram analisadas conforme a sequência de evocação das unidades de registro. Desta forma, mães e pais que declararam quatro metas de desenvolvimento emocional para seus filhos, por exemplo, tiveram tais metas consideradas como quatro unidades de registro, e analisadas como 'primeira evocação', 'segunda evocação', 'terceira evocação', e assim por diante. Todas as análises foram realizadas com o software estatístico SPSS for Windows, versão 20.0.

\section{Resultados}

\section{Características emocionais desejadas para os filhos}

As categorias automaximização e outras possibilidades (metas declaradas não passíveis de classificação) apresentaram as maiores frequências entre as primeiras, segundas e terceiras evocações de metas nas respostas de mães e pais. Na primeira evocação, a automaximização esteve presente em $50,8 \%(n=61)$ das metas relatadas, seguida por metas não passíveis de classificação, relativas a $35,8 \%(n=43)$. Na segunda e terceira evocações, metas não passíveis de classificação foram as mais evocadas (com $46,5 \%$ e $52,1 \%$, respectivamente), seguidas por metas de automaximização (com $37,4 \%$ e $29,6 \%$, respectivamente). O teste qui-quadrado de aderência indicou que a distribuição das categorias não se deu de forma homogênea, havendo preponderância estatisticamente significativa de uma ou mais categorias em detrimento de outras nas metas presentes na primeira $\left(X^{2}{ }_{3}=70,20 ; p<0,05\right)$, segunda $\left(X^{2}{ }_{3}=\right.$ $47,06 ; p<0,05)$ e terceira evocações $\left(X^{2}{ }_{3}=36,44 ; p<0,05\right)$.

Não foram encontradas diferenças significativas entre mães e pais na evocação das características emocionais que desejavam para seus filhos. Tal resultado foi válido para as metas declaradas na primeira $\left(x^{2}{ }_{3}=3,69 ; p>0,05\right)$, segunda $\left(x^{2}{ }_{3}=4,04 ; p>0,05\right)$ e terceira $\left(x^{2}{ }_{3}=\right.$ $2,88 ; p>0,05)$ evocações. Pelo resultado do teste, entendeu-se que aquilo que mães e pais declararam quanto a características emocionais que desejavam para seus filhos não difere, de forma estatisticamente significativa, quanto à evocação das categorias. 
Na comparação de mães e pais de meninos VS mães e pais de meninas, o teste qui-quadrado de associação também não indicou diferenças significativas quanto às características que desejavam para seus filhos. O mesmo foi válido para as metas declaradas na primeira $\left(x^{2}{ }_{3}=1,92 ; p>0,05\right)$, segunda $\left(x^{2}{ }_{3}=0,82 ; p>0,05\right)$ e terceira $\left(x^{2}{ }_{3}=\right.$ $1,34 ; p>0,05)$ evocações.

\section{Condições necessárias para o desenvolvimento emocional dos filhos}

Nas respostas de mães e pais acerca das condições necessárias para que seus filhos desenvolvam ou venham a ter as características emocionais desejadas, a categoria de maior frequência em todas as evocações foi a de condições centradas no contexto. Presente em $51,3 \% \quad(n=61)$ das primeiras evocações, $50,7 \% \quad(n=38)$ das segundas e $41,2 \%(n=14)$ das terceiras. A categoria com a segunda maior frequência nas respostas foi a de centradas na mãe/cuidador, presente em $17,6 \%(n=21)$ das primeiras evocações, $20 \%(n=15)$ das segundas evocações, e 32,4\% $(n=11)$ das terceiras evocações. O teste qui-quadrado de aderência não revelou homogeneidade na distribuição das categorias nas evocações de mães e pais, indicando preponderância de certas categorias em detrimento de outras. Tal resultado foi válido para a primeira $\left(x^{2}{ }_{4}=83,48 ; p<0,05\right)$, segunda $\left(x^{2}{ }_{3}=27,35 ; p<0,05\right)$ e terceira $\left(x^{2}{ }_{3}=8,59 ; p<0,05\right)$ evocações.

$\mathrm{Na}$ comparação de mães vs pais quanto às condições necessárias para o desenvolvimento das características emocionais que desejam para seus filhos, o teste qui-quadrado de associação não indicou diferenças significativas entre as respostas de mães e pais quanto às categorias da primeira evocação $\left(X^{2}{ }_{4}=0,82 ; p>0,05\right)$ e da segunda evocação $\left(X^{2}{ }_{3}=0,71 ; p>0,05\right)$. Não foi possível realizar o teste para terceira evocação de categorias, devido ao número elevado de células com valor inferior a cinco.

De igual modo, não foram encontradas diferenças significativas entre as categorias presentes nas condições indicadas por mães e pais de meninos VS mães e pais de meninas. O teste qui-quadrado de associação não indicou diferenças para as respostas da primeira evocação $\left(X^{2}{ }_{4}=2,98 ; p>0,05\right)$, da segunda $\left(X^{2}{ }_{3}=2,13 ; p>0,05\right)$ ou da terceira $\left(X^{2}{ }_{3}=0,24 ; p>0,05\right)$.

\section{Atuação parental necessária para o desenvolvimento das características emocionais desejadas para os filhos}

Acerca da participação parental necessária para o desenvolvimento das características que desejavam para seus filhos, a categoria mais frequente nas respostas das mães e pais foi a de educar/orientar, presente em 59,2\% $(n=71)$ das falas na primeira evocação, $49,4 \%$ 
( $n=38)$ na segunda evocação e $50 \%(n=22)$ na terceira evocação. 0 teste de significância estatística, indicado pelo qui-quadrado de aderência, apontou para a não homogeneidade na distribuição das categorias para primeira $\left(X^{2}{ }_{4}=133,67 ; p<0,05\right)$, segunda $\left(X^{2}{ }_{3}=\right.$ $27,26 ; p<0,05)$ e terceira $\left(X^{2}{ }_{3}=15,82 ; p<0,05\right)$ evocações.

Mães e pais não diferiram quanto à evocação das categorias referentes à sua atuação para o desenvolvimento das características emocionais que desejavam para os filhos. O teste qui-quadrado não indicou diferenças significativas entre as respostas de mães e pais quanto às categorias da primeira evocação $\left(X^{2}{ }_{4}=3,00 ; p>0,05\right)$ e da segunda evocação $\left(X^{2}{ }_{3}=1,92 ; p>0,05\right)$. Não foi possível realizar 0 teste para terceira evocação de categorias, devido ao número elevado de células com valor inferior a cinco.

Analisando se havia diferença entre mães e pais de meninos VS mães e pais de meninas, o teste qui-quadrado de associação também não indicou diferenças significativas na evocação das categorias para a primeira $\left(X^{2}{ }_{4}=5,39 ; p>0,05\right)$ e segunda evocações $\left(X^{2}{ }_{3}=1,26\right.$; $p>0,05)$. O teste para a terceira evocação não pôde ser realizado devido ao número elevado de células com valor esperado inferior a cinco.

Uma síntese dos principais resultados é apresentada na Tabela 1.

Tabela 1

Sintese Principais Resultados

\begin{tabular}{|c|c|c|c|}
\hline & $\begin{array}{l}\text { Categoria com } \\
\text { maior frequência } \\
\left(1^{a} \text { evocação) }\right.\end{array}$ & $\begin{array}{c}\text { Dif. entre mãe e } \\
\text { pai } \\
\left(1^{a} \text { evocação. }\right)\end{array}$ & $\begin{array}{c}\text { Dif. sexo da } \\
\text { criança } \\
\left(1^{a} \text { evocação) }\right.\end{array}$ \\
\hline $\begin{array}{l}\text { Características } \\
\text { emocionais desejadas } \\
\text { para os filhos }\end{array}$ & $\begin{array}{l}\text { Automaximização } \\
50,8 \%(n=61) \text { das } \\
\text { metas enunciadas }\end{array}$ & $\begin{array}{c}\text { Não } \\
\left(x^{2} 3=3,69 ; p>\right. \\
0,05)\end{array}$ & $\begin{array}{c}\text { Não } \\
\left(x^{2} 3=1,92 ; p>\right. \\
0,05)\end{array}$ \\
\hline $\begin{array}{l}\text { Condições necessárias } \\
\text { para o } \\
\text { desenvolvimento } \\
\text { emocional dos filhos }\end{array}$ & $\begin{array}{c}\text { Centradas no } \\
\text { contexto } \\
51,3 \%(n=61) \text { das } \\
\text { metas enunciadas }\end{array}$ & $\begin{array}{c}\text { Não } \\
\left(X^{2} 4=0,82\right. \\
p>0,05)\end{array}$ & $\begin{array}{c}\text { Não } \\
\left(X^{2} 4=2,98\right. \\
p>0,05)\end{array}$ \\
\hline $\begin{array}{l}\text { Atuação parental para } \\
\text { o desenvolvimento das } \\
\text { características } \\
\text { desejadas }\end{array}$ & $\begin{array}{c}\text { Educar/orientar } \\
59,2 \%(n=71) \text { das } \\
\text { metas enunciadas }\end{array}$ & $\begin{array}{c}\text { Não } \\
\left(x^{2} 4=3,00 ;\right. \\
p>0,05)\end{array}$ & $\begin{array}{c}\text { Não } \\
\left(X^{2} 4=5,39\right. \\
p>0,05)\end{array}$ \\
\hline
\end{tabular}

\section{Discussão}

As análises dos dados indicaram que, considerando-se todo 0 conjunto de participantes do estudo, as concepções parentais a respeito das características emocionais desejadas para os filhos quando adultos são indicativas de uma valorização da autonomia, 
pela preponderância da categoria de automaximização. Como apontado por Leyendecker et al. (2002), uma valorização maior da independência e autonomia na criança é refletida em maior frequência de respostas que se referem ao desenvolvimento nos planos pessoal, profissional e interpessoal, incluindo bem-estar emocional e independência psicológica, ideias que remetem à categoria aqui definida como automaximização.

Ao serem investigadas metas de socialização em mães brasileiras foi verificada uma orientação autônomo-relacionada (Seidl-de-Moura et al., 2008), sendo que mães do Rio de Janeiro indicaram tendência à valorização da autonomia, mas com preocupações também de natureza sociocêntrica (Seidl-de-Moura et al., 2009). O que se pode depreender dos resultados encontrados nesse estudo voltado para as metas de emoção, é que as mães e pais do Rio de Janeiro que participaram da pesquisa privilegiaram a autonomia nesse domínio específico do desenvolvimento, envolvendo demonstrações de segurança e autoconfiança no lidar com as emoções e ao expressálas.

Em relatos de Fonseca et al. (2017), igualmente voltados para metas de socialização da emoção, mães de um contexto rural brasileiro, também enunciaram majoritariamente metas associadas à automaximização, mas ressaltaram ainda a preocupação em que a criança desenvolvesse a capacidade para a intimidade emocional com outras pessoas. Para as autoras, os resultados sugerem que 0 contexto está passando por uma transição com mudança gradual para uma orientação de self autônomo-relacionada. De todo modo, os resultados aqui relatados mostram que em certos ambientes de grande urbanização como o Rio de Janeiro, as metas de emoção podem tender nitidamente para autonomia. Certas características dos participantes, como o nível de escolaridade, também podem contribuir para os resultados, ressaltando-se que a grande maioria das mães e pais dessa investigação tinham nível superior completo.

Desejos de que o filho quando adulto tenha capacidade de regulação emocional e saiba manter relacionamentos afetivos não foram marcantes entre as mães e pais participantes dessa pesquisa. Assim, não parece haver maior preocupação em que desenvolvam características emocionais voltadas para maior proximidade interpessoal. Pode-se especular, acompanhando a argumentação de Vieira et al. (2010), que esse resultado possivelmente esteja associado ao elevado grau de urbanização do contexto estudado, vinculado a aumento da competitividade e diminuição de valores ligados ao grupo social.

Marca os resultados encontrados uma predominância que se mantém quando contrastados o grupo de mães com o de pais, e o grupo de mães e pais de meninas com o de mães e pais de meninos. Ao que parece, a valorização de características emocionais vinculadas à 
maior autonomia independe de ser um filho ou uma filha e parece ser um desejo compartilhado pela dupla de pais. Neste aspecto, entretanto, é sentida a ausência de investigações que forneçam resultados que possam ser contrastados com os encontrados.

Cabe observar ainda a elevada frequência de respostas que não puderam ser classificadas em uma das categorias temáticas de interesse do estudo e ficaram alocadas à categoria outras possibilidades. Pode-se especular que o sistema de categorização proposto não tenha sido abrangente o suficiente e carece de revisão, ou que as metas para o desenvolvimento emocional dos filhos na amostra foram muito heterogêneas, dificultando a identificação de um padrão homogêneo. É possível considerar ainda que os participantes tenham encontrado dificuldade em formular com clareza cognições a respeito dessa temática envolvendo emoções.

A preponderância da categoria centradas no contexto quanto às condições necessárias para que os filhos desenvolvam as características emocionais desejadas aponta para a relevância atribuída por mães e pais à possibilidade de oferecerem a suas crianças boas oportunidades sociais, provendo educação de qualidade e criando-as em um ambiente familiar saudável. Esse resultado se afasta do encontrado por Fonseca et al. (2017), com mães de um contexto rural que viam a si mesmas como a fonte principal de provimento aos filhos das condições para que se desenvolvam do modo esperado. Tal contraste pode ser pensado em termos das diferenças entre contextos socioculturais, e de diferentes níveis de urbanização (Vieira et al., 2010). Mães e pais do Rio de Janeiro, tenham filhos ou filhas, tenderam a manifestar essa concepção sinalizando que a variável sexo, para os pais ou para os filhos, não implica diferença quanto ao que pensam. Também com relação a esse foco de interesse, não foram encontradas evidências na literatura que permitam ampliar a discussão.

Ao ser analisado o tipo de atuação parental para o desenvolvimento de características emocionais desejadas, houve preponderância da categoria educar/orientar. Mais uma vez, encontrou-se consonância quanto a isto, tanto na comparação de grupo de mães e de pais, quanto confrontando o grupo de mães e pais de meninas e de meninos. Os achados do presente estudo, mesmo com uma amostra de mães e pais de contexto urbano, se assemelham aos achados de Fonseca et al. (2017) com cuidadores de contexto rural, que também indicaram, preponderantemente, atividades próprias do educar e orientar os filhos como parte essencial do papel que thes cabe no desenvolvimento emocional de suas crianças. Ao que parece, diferenças socioculturais e de nível de urbanização entre os contextos não seriam marcantes para o estabelecimento de formas distintas de mães e pais pensarem a esse respeito. 
Em síntese, pode-se considerar que as metas parentais de socialização de emoção encontradas apontaram para uma maior valorização de metas voltadas para a autonomia, estando em consonância com o encontrado em outros ambientes urbano sem que o nível médio de escolaridade não era baixo. Em famílias da classe média urbana ocidental de Berlim, Keller (2016) e Keller e Otto (2009) mencionam tendência equivalente e em Hong Kong, ainda que priorizando metas relacionais, as mães também enfatizaram a competência emocional individualista como meta de desenvolvimento dos filhos (Chan, 2011).

Quanto às condições necessárias para o desenvolvimento emocional dos filhos, estas foram associadas, sobretudo, ao que pode ser obtido ou é oferecido pelo contexto, diferentemente do encontrado em contexto rural brasileiro (Fonseca et al., 2017), em que as mães priorizaram condições centradas nelas mesmas (ou outros cuidadores primários). Já no que concerne à atuação parental, práticas educativas e de orientação foram as que sobressaíram, tal como relatado para este mesmo contexto rural brasileiro. Metas concebidas por mães e pais convergiram e foram independentes de terem filhos ou filhas.

\section{Considerações finais}

O propósito desse estudo foi o de examinar metas de socialização de emoções em mães e pais de crianças de até três anos, residentes na cidade do Rio de Janeiro, e analisar se haveria diferenças nas metas estabelecidas por mães e por pais, assim como nas metas estabelecidas para filhas e filhos. Nesse sentido, entende-se que essa investigação alcançou seu propósito, trazendo contribuições, uma vez que foram caracterizadas as metas de socialização voltadas para emoção traçadas por mães e pais do contexto investigado, bem como contrastadas as metas evocadas por mães e pais, e por mães-pais de filhos e filhas, encontrando-se confluência de resultados nessas comparações.

A convergência de metas e ideias relatadas pelos participantes pode ser indicativa de certa homogeneidade ou harmonia de pensamento e objetivos entre mães e pais, independentemente do sexo dos filhos, bem como de um viés amostral. Não se pode deixar de conjecturar que pais e mães que se disponham a participar de uma pesquisa com esse objeto de interesse, refletindo e falando sobre expectativas ligadas a emoções, caracterizem-se pelo compartilhamento de convicções e metas de desenvolvimento para seus filhos.

Ainda que se entenda que a presente investigação tenha trazido contribuições ao campo de estudos dos sistemas parentais e desenvolvimento emocional infantil, seus resultados devem ser 
tomados com a devida precaução, dadas algumas limitações da pesquisa realizada. A amostra foi composta somente por famílias de configuração nuclear, com alto nível de escolaridade e em um contexto urbano do Rio de Janeiro.

Considerando o presente trabalho como um esforço exploratório, no sentido de explicitar as metas de socialização de emoções de um grupo específico, indicamos a necessidade de estudos com amostras envolvendo distintas configurações familiares (ex.: monoparentais, reconstituídas), com grupos de participantes de variados níveis de escolaridade e em outros contextos socioculturais, a fim de avançar no conhecimento acerca dessas metas em contextos brasileiros. De todo modo, tendo em vista que muito poucos foram os estudos identificados na literatura científica, e destes apenas um brasileiro, acredita-se que uma contribuição expressiva foi trazida.

\section{Referências}

Chan, S. M. (2011). Mothers' construal of self and emotion socialisation goals. Early Education \& Development, 181(5), 613-624. doi: 10.1080/03004431003671820

Fonseca, B. R., Cavalcante, L. I., \& Mendes, D. M. L. F. (2017). Metas de socialização da emoção: Um estudo de mães residentes no meio rural. PSICO, 48(3), 174-185. doi:10.15448/19808623.2017.3.25444

Harwood, R. L., Schölmerich, A., Ventura-Cook, E., Schulze, P. A., \& Wilson, S. P. (1996). Culture and class influences on Anglo and Puerto Rican mothers' beliefs regarding long-term socialization goals and child behavior. Child Development, 67, 2446-2461.

Kağitçibaşi, Ç. (2007). Family, self, and human development across cultures: Theory and applications. Mahwah, NJ: Lawrence Erlbaum Associates.

Keller, H. (2016). Psychological autonomy and hierarchical relatedness as organizers of developmental pathways. Philosophical Transactions of the Royal Society B, 371(1686), 1-9. doi: $10.1098 /$ rstb. 2015.0070

Keller, H., \& Kärtner, J. (2013). Development: The Cultural Solution of Universal Developmental Tasks. In M. J. Gelfand, C. Chiu, \& Y. Hong (Eds.), Advances in Culture and Psychology (pp. 63-116). New York: Oxford Press.

Keller, H., \& Otto, H. (2009). The cultural socialization of emotion regulation during infancy. Journal of Cross-Cultural Psychology, 40(6), 996-1011. doi:10.1177/0022022109348576

Leyendecker, B., Lamb, M. E., Harwood, R. L., \& Schölmerich, A. (2002). Mothers' socialization goals and evaluation of desirable and undesirable everyday situations in two diverse cultural 
groups. International Journal of Behavioral Development, 26(3), 248-258. doi:10.1080/01650250143000030

Mendes, D. M. L. F. Pessôa, L. F., \& Cavalcante, L. I. C. (2016). Metas parentais de socialização da emoção e modelos de self: Uma articulação conceitual. Estudos e Pesquisas em Psicologia, 16(2), 450-468. doi:10.12957/epp.2016.29171

Oliveira, D. C. (2008). Análise de conteúdo temático-categorial: Uma proposta de sistematização. Revista de enfermagem UERJ, 16(4), 569-76. Recuperado de http://www.facenf.uerj.br/v16n4/v16n4a19.pdf

Otto, H., \& Keller, H. (2016). Is there something like German parenting? In G. Nicolas, A. Bejarano, \& D. L. Lee (Eds.), Contemporary Parenting: a Global Perspective (pp. 81-94). New York: Routledge/Taylor \& Francis Group.

Park, H., Coello, J. A., \& Lau, A. S. (2014). Child Socialization Goals in East Asian versus Western Nations from 1989 to 2010: Evidence for Social Change in Parenting. Parenting: Science and Practice, 14(2), 69-91. doi:10.1080/15295192.2014.914345

Raval, V. V., Raval, P. H., \& Deo, N. (2014). Mothers' Socialization Goals, Mothers' Emotion Socialization Behaviors, Child Emotion Regulation, and Child Socioemotional Functioning in Urban India. Journal of Early Adolescence, 34(2), 229-250. doi:10.1177/0272431613485821

Seidl-de-Moura, M. L., Lordelo, E. R., Vieira, M. L., Piccinini, C. A., Siqueira, J. O., Magalhães, C. M. C., Pontes, F. A. R., Salomão, N. M., Rimoli, A. O. (2008). Brazilian mothers' socialization goals: Intracultural differences in seven Brazilian cities. International Journal of Behavioral Development, 32(6), 480487. doi: $10.1177 / 0165025408093666$

Seidl-de-Moura, M. L., Targino, T., Nahum, K., Cruz, E. M., Amaral, G., \& Marca, R. (2009). Parenting cultural models of a group of mothers from Rio de Janeiro, Brazil. The Spanish Journal of Psychology, 12(2), 506-517. doi:10.1017/S1138741600001888

Vieira, M. L., Seidl-de-Moura, M. L., Mafioletti, S. M., Martins, G. D. F., Tokumaru, R. S., Lordelo, E., \& Keller, H. (2010). Autonomy and interdependence: Beliefs of Brazilian mothers from State capitals and small towns. The Spanish Journal of Psychology, 13(2), 816-824. doi:10.1017/S113874160000247X

Sen H., Yavuz-Muren H. M., \& Yagmurlu, B. (2014). Parenting: The Turkish Context. In H. Selin (Ed.), Parenting Across Cultures: Childrearing, Motherhood and Fatherhood in Non-Western Cultures (pp. 175-192). Dordrecht, Netherlands: Springer.

Tamm, A., Kasearu, K., Tulviste, T., \& Trommsdorff, G. (2016). Maternal values and parenting and Estonian, German, and Russian adolescents' friendship satisfaction. Personal Relationships, 23(2), 249-264. doi:10.1111/pere.12123 


\section{Endereço para correspondência \\ Deise Maria Leal Fernandes Mendes}

Universidade do Estado do Rio de Janeiro

Rua São Francisco Xavier, 524, $10^{\circ}$ andar, sala 10.004F, Maracanã, CEP 20550-990, Rio de Janeiro - RJ, Brasil

Endereço eletrônico: deisefmendes@gmail.com

\section{Joana Lezan Sant'Anna}

Universidade do Estado do Rio de Janeiro

Rua São Francisco Xavier, 524, $10^{\circ}$ andar, sala 10.004F, Maracanã, CEP 20550-990, Rio de Janeiro - RJ, Brasil

Endereço eletrônico: jolezan@gmail.com

\section{Dandara de Oliveira Ramos}

Instituto Gonçalo Moniz

Edifício Tecnocentro

Rua Mundo, 121, Trobogy, CEP 41745-715, Salvador - BA, Brasil

Endereço eletrônico: dandararamos2@gmail.com

Recebido em: 31/03/2019

Reformulado em: 12/10/2019

Aceito em: 24/10/2019

\section{Notas}

* Doutorado em Psicologia Social. Professora Adjunta do Instituto de Psicologia da Universidade do Estado do Rio de Janeiro.

** Mestrado em Psicologia Social. Doutoranda do Programa de Pós-Graduação em Psicologia Social da Universidade do Estado do Rio de Janeiro.

*** Doutorado em Saúde Coletiva. Pós-Doutoranda do Centro de Integração de Dados e Conhecimentos para a Saúde - Instituto Gonçalo Moniz, Fiocruz-Bahia.

Este artigo de revista Estudos e Pesquisas em Psicologia é licenciado sob uma Licença Creative Commons Atribuição-Não Comercial 3.0 Não Adaptada. 